\title{
Critical nonlinear Schrödinger equations in higher space dimensions
}

\author{
By Nakao Hayashi, Chunhua Li and Pavel I. NAumkin
}

(Received Jan. 16, 2017)

(Revised Apr. 13, 2017)

\begin{abstract}
We study the critical nonlinear Schrödinger equations

$$
i \partial_{t} u+\frac{1}{2} \Delta u=\lambda|u|^{2 / n} u, \quad(t, x) \in \mathbb{R}^{+} \times \mathbb{R}^{n},
$$

in space dimensions $n \geq 4$, where $\lambda \in \mathbb{R}$. We prove the global in time existence of solutions to the Cauchy problem under the assumption that the absolute value of Fourier transform of the initial data is bounded below by a positive constant. Also we prove the two side sharp time decay estimates of solutions in the uniform norm.
\end{abstract}

\section{Introduction and main results.}

We consider the initial value problem for the following nonlinear Schrödinger equation

$$
\left\{\begin{array}{l}
i \partial_{t} u+\frac{1}{2} \Delta u=\lambda|u|^{2 / n} u, \quad(t, x) \in \mathbb{R}^{+} \times \mathbb{R}^{n}, \\
u(0, x)=u_{0}(x), \quad x \in \mathbb{R}^{n}
\end{array}\right.
$$

in space dimensions $n \geq 4$, where $\lambda \in \mathbb{R}$. In the case of $1 \leq n \leq 3$, asymptotic behavior of small amplitude solutions to (1.1) has been studied in $[\mathbf{2}],[\mathbf{5}],[\mathbf{7}],[\mathbf{8}],[\mathbf{1 4}]$ and etc. The first breakthrough in asymptotic behavior of small solutions to (1.1) with $n=1$ was obtained in [14] by introducing the final state

$$
e^{i|x|^{2} / 2 t-i(\pi / 4)} t^{-1 / 2} e^{-i \lambda\left|\widehat{u_{+}}(x / t)\right|^{2} \log t} \widehat{u_{+}}\left(\frac{x}{t}\right)
$$

for given small final data $\widehat{u_{+}}$. More precisely, existence of small solutions was shown in the neighborhood of final state to define the map $W_{+}: \widehat{u_{+}} \in \mathbf{H}^{3,0} \cap \mathbf{H}^{2,1} \rightarrow u(0) \in \mathbf{L}^{2}$, where

$$
\mathbf{H}^{m, s}=\left\{\phi \in \mathbf{L}^{2} ;\left\|(1-\Delta)^{m / 2}\left(1+|x|^{2}\right)^{s / 2} \phi\right\|_{\mathbf{L}^{2}}<\infty\right\}
$$

2010 Mathematics Subject Classification. Primary 35Q55, 35B40.

Key Words and Phrases. critical NLS equations, higher space dimensions, large time asymptotics.

The firtst author was partially supported by JSPS KAKENHI Grant Numbers JP25220702, JP15H03630. The second author was partially supported by NNSFC Grant No.11461074. The third author was partially supported by CONACYT and PAPIIT project IN100616. 
This result was extended in $n=2,3$ in [5] by modifying the phase corrections. In [2], the result concerning the regularity of solutions in [14] was improved as the map $W_{+}: \widehat{u_{+}} \in \mathbf{H}^{3,0} \cap \mathbf{H}^{2,1} \rightarrow u(0) \in \mathbf{H}^{0,1} \cap \mathbf{H}^{1,0}$. On the other hand, the initial value problem was treated in [7] and existence and uniqueness of the scattering data $u_{-}$was shown for any small initial data $\widehat{u_{0}} \in \mathbf{H}^{0, \delta} \cap \mathbf{H}^{\delta, 0}, n / 2<\delta<1+2 / n$. Therefore the map $W_{-}^{-1}: \widehat{u_{0}} \in \mathbf{H}^{0, \delta} \cap \mathbf{H}^{\delta, 0} \rightarrow \widehat{u_{-}} \in \mathbf{L}^{2}$ is defined. This fact with the result of [2] means that the map $W_{-}^{-1} W_{+}: \widehat{u_{+}} \in \mathbf{H}^{3,0} \cap \mathbf{H}^{2,1} \rightarrow \widehat{u_{-}} \in \mathbf{L}^{2}$ is defined in the case of $n=1$. In [8], by using another final state such that

$$
\mathcal{F} e^{i\left(|\xi|^{2} / 2\right) t} \widehat{u_{+}}(\xi) e^{-i \lambda\left|\widehat{u_{+}}(\xi)\right|^{2} \log t}
$$

the results mentioned in the above were improved as $W_{-}^{-1} W_{+}: \widehat{u_{+}} \in \mathbf{H}^{\alpha, 0} \rightarrow \widehat{u_{-}} \in \mathbf{H}^{\delta, 0}$, where

$$
\frac{n}{2}<\delta<\alpha<\min \left\{n, 2,1+\frac{n}{2}\right\}
$$

which enables us to define the inverse operator of $W_{-}^{-1} W_{+}$. This inequality requires $n \leq 3$. However as far as we know, there are no results in the case of $n \geq 4$ even if the existence of $W_{+}$. Our purpose in this paper is to show the sharp asymptotics and time decay of solutions to (1.1) in the uniform norm for higher space dimensions $n \geq 4$. Our second result below shows that existence of the map

$$
W_{+}^{-1}: e^{(i / 2)|\xi|^{2}} \widehat{u_{0}} \in \mathbf{L}^{\infty} \cap \dot{\mathbf{H}}^{\sigma, 0} \rightarrow \widehat{u_{+}} \in \mathbf{L}^{\infty} \cap \dot{\mathbf{H}}^{\beta, 0}, \quad \frac{n}{2}<\beta<\sigma<\frac{n}{2}+1 .
$$

By the factor $e^{(i / 2)|\xi|^{2}}$, we shift $t=0$ to $t=1$, then we do not know existence of the operator $W_{-}^{-1} W_{+}$.

Cubic nonlinear Klein-Gordon equation is considered as a relativistic version of cubic nonlinear Schrödinger equation and asymptotic behavior of small solutions was studied in $[\mathbf{4}],[\mathbf{9}]$ and $[\mathbf{1 0}]$. Recently, in [13] final state problem was solved for the nonlinearity $|u| u$. However the Cauchy problem in higher space dimensions is still an open problem. Thus scattering problem is not developed in the case of nonlinear Klein-Gordon equation as compared with the case of nonlinear Schrödinger equation.

As in the proof of [7], we multiply both sides of (1.1) by $\mathcal{F U}(-t)$ to obtain

$$
i \partial_{t} \mathcal{F U}(-t) u=\lambda t^{-1} \mathcal{F} M(-t) \mathcal{F}^{-1}|\mathcal{F} M(t) \mathcal{U}(-t) u|^{2 / n} \mathcal{F} M(t) \mathcal{U}(-t) u,
$$

where $\mathcal{U}(t)$ is the Schrödinger evolution group, $M(t)=e^{i|x|^{2} / 2 t}$ for $t \neq 0$,

$$
\left(D_{t} \phi\right)(x)=\frac{1}{(i t)^{n / 2}} \phi\left(\frac{x}{t}\right)
$$

is the dilation operator, $\mathcal{F}$ denotes the Fourier transformation, where we have used the formulas $\mathcal{U}(t)=M(t) D_{t} \mathcal{F} M(t)$ and $\mathcal{U}(-t)=M(-t) \mathcal{F}^{-1} D_{t}^{-1} M(-t)$. We decompose the nonlinear term of the above equation into the main term

$$
\lambda t^{-1}|\mathcal{F U}(-t) u|^{2 / n} \mathcal{F U}(-t) u
$$


and the remainder term $R$, then we have the ordinary differential equation

$$
i \partial_{t} \mathcal{F U}(-t) u=\lambda t^{-1}|\mathcal{F U}(-t) u|^{2 / n} \mathcal{F U}(-t) u+R .
$$

The difficulty comes from the lack of regularity of the first term on the right-hand side of the above equation. To avoid this difficulty, we consider the problem in the closed subset of the function space satisfying the restriction $|\mathcal{F U}(-t) u| \neq 0$. Therefore we do not consider the problem in the space $\mathcal{F U}(-t) u \in \mathbf{L}^{p}, 1<p<\infty$. Previous works for (1.1) were based on $\mathbf{H}^{s}$ space for $\mathcal{F U}(-t) u$, where $s>n / 2$. Function spaces which do not necessarily include $\mathbf{L}^{2}$ were used in [12], [6].

We introduce some function spaces and notations. Let $\mathbf{L}^{\infty}$ denote the usual Lebesgue space with the norm $\|\phi\|_{\mathbf{L}^{\infty}}=$ ess. $\sup _{x \in \mathbb{R}^{n}}|\phi(x)|$. The homogeneous Sobolev space $\dot{\mathbf{H}}_{r}^{m}$ is defined by

$$
\dot{\mathbf{H}}_{r}^{m}=\left\{\phi ;\|\phi\|_{\dot{\mathbf{H}}_{r}^{m}}=\left\|(-\Delta)^{m / 2} \phi\right\|_{\mathbf{L}^{r}}<\infty\right\},
$$

$m \geq 0$, where $\|\phi\|_{\mathbf{L}^{r}}^{r}=\int_{\mathbb{R}^{n}}|\phi(x)|^{r} d x$. Denote $\langle t\rangle=\sqrt{1+t^{2}}$. For simplicity, we write $\dot{\mathbf{H}}_{2}^{m}=\dot{\mathbf{H}}^{m}$. By $\dot{\mathbf{B}}_{p, q}^{s}$ we denote the homogeneous Besov space with semi-norm

$$
\|\phi\|_{\dot{\mathbf{B}}_{p, q}^{s}}=\left(\int_{0}^{\infty} x^{-1-\gamma q} \sup _{|y| \leq x} \sum_{|\theta|=[s]}\left\|\partial^{\theta}\left(\phi_{y}-\phi\right)\right\|_{\mathbf{L}^{p}}^{q} d x\right)^{1 / q}
$$

where $s=[s]+\gamma, 0<\gamma<1, \phi_{y}(x)=\phi(x+y), 1 \leq p, q \leq \infty$ and $[s]$ is the largest integer less than $s$. Different positive constants might be denoted by the same letter $C$ if it does not cause any confusion.

To state our results, we use the function space

$$
\mathbf{X}=\left\{u ; \mathcal{F U}(-t-1) u \in \mathbf{C}([0, \infty) ; \mathbf{Y}),\|u\|_{\mathbf{X}}<\infty\right\}
$$

where $\mathbf{Y}=\mathbf{L}^{\infty} \cap \dot{\mathbf{H}}^{\sigma}, n / 2<\sigma<n / 2+2$ and

$$
\|u\|_{\mathbf{X}}=\sup _{0 \leq t<\infty}\|\mathcal{F U}(-t-1) u(t)\|_{\mathbf{L}^{\infty}}+(t+1)^{-\gamma}\|\mathcal{F U}(-t-1) u(t)\|_{\dot{\mathbf{H}}^{\sigma}}
$$

with a small $\gamma$ satisfying $(1 / n)(\sigma-n / 2)>\gamma>0$. We note here that the Hölder class of order $\sigma-n / 2$ is included in $\mathbf{Y}$.

THEOREM 1.1. We assume that the initial data satisfy

$$
\frac{\rho}{2} \leq \inf _{\xi \in \mathbb{R}^{n}}\left|\widehat{u_{0}}(\xi)\right| \leq\left\|\widehat{u_{0}}\right\|_{\mathbf{L}^{\infty}} \leq \rho
$$

and $\left\|e^{(i / 2)|\xi|^{2}} \widehat{u_{0}}\right\|_{\dot{\mathbf{H}}^{\sigma}} \leq \rho^{2}$ with $n / 2<\sigma<n / 2+1$. Then there exists a $\rho_{0}>0$ such that the Cauchy problem (1.1) has a unique solution $u \in \mathbf{X}$ for all $0<\rho \leq \rho_{0}$. Moreover the time decay estimate

$$
\frac{1}{5} \rho(t+1)^{-n / 2} \leq \inf _{x \in \mathbb{R}^{n}}|u(t)| \leq\|u(t)\|_{\mathbf{L}^{\infty}} \leq \frac{7}{5} \rho(t+1)^{-n / 2}
$$


holds for all $t>0$.

REMARK 1.1. Typical example of the data could be the following

$$
\widehat{u_{0}}(\xi)=e^{(-i / 2)|\xi|^{2}} \rho\left(1-\frac{\rho^{2}}{\langle\xi\rangle}\right)
$$

since by a direct calculation

$$
\left\|e^{(i / 2)|\xi|^{2}} \widehat{u_{0}}\right\|_{\dot{\mathbf{H}}^{\sigma}}=\left\|\rho\left(1-\frac{\rho^{2}}{\langle\xi\rangle}\right)\right\|_{\dot{\mathbf{H}}^{\sigma}} \leq C \rho^{3} \leq \rho^{2}
$$

and

$$
\rho-\rho^{3} \leq \inf _{\xi \in \mathbb{R}^{n}}\left|\widehat{u_{0}}(\xi)\right| \leq\left\|\widehat{u_{0}}\right\|_{\mathbf{L} \infty} \leq \rho .
$$

TheOREM 1.2. $\quad$ Let $u$ be the solution constructed in Theorem 1.1. Then there exists a unique final state $\widehat{u_{+}} \in \mathbf{L}^{\infty} \cap \dot{\mathbf{H}}^{\beta}, n / 2<\beta<\sigma<n / 2+1$, such that the asymptotics

$$
\begin{gathered}
\left\|u(t)-e^{i|x|^{2} / 2(t+1)-i(n \pi / 4)}(t+1)^{-n / 2} e^{-i \lambda\left|\widehat{u_{+}}(x /(t+1))\right|^{2 / n} \log (t+1)} \widehat{u_{+}}\left(\frac{x}{t+1}\right)\right\|_{\mathbf{L}^{\infty}} \\
\leq C(t+1)^{-n / 2-(2 / n)(\delta-\gamma)}\left(\rho^{2}+\rho^{(2 / n+2)(2 / n)+1} \log (t+1)\right)
\end{gathered}
$$

and

$$
\begin{array}{r}
\left\|(\mathcal{F U}(-t-1) u)(t)-\widehat{u_{+}} e^{-i \lambda\left|\widehat{u_{+}}\right|^{2 / n} \log (t+1)}\right\|_{\dot{\mathbf{H}}^{\beta}} \\
\leq C \rho^{4 / n+2}(t+1)^{(-1 / 2)(\sigma-\beta)+\gamma} \log (t+1)
\end{array}
$$

hold for all $t>0$. Furthermore we have

$$
\frac{1}{5} \rho \leq \inf _{\xi \in \mathbb{R}^{n}}\left|\widehat{u_{+}}(\xi)\right| \leq\left\|\widehat{u_{+}}\right\|_{\mathbf{L}^{\infty}} \leq \frac{7}{5} \rho,
$$

where $\delta \in(0,(1 / 2)(\sigma-n / 2)), 0<\gamma<(\sigma-\beta) / n$.

To explain our results, we look for the solution of (1.1) in the form

$$
u(t, x)=(i(t+1))^{-n / 2} e^{i|x|^{2} / 2(t+1)} h(t), \quad h(0)=\rho .
$$

By a direct calculation, $h(t)$ satisfies the ordinary differential equation

$$
i h^{\prime}=\lambda(t+1)^{-1}|h|^{2 / n} h .
$$

We change the dependent variable $h=r e^{i w}, r=|h|, w=\arg h$, with $r(0)=\rho>0$, $w(0)=0$, then we have

$$
i r^{\prime}-r w^{\prime}=\lambda(t+1)^{-1} r^{2 / n+1},
$$

which gives us the ordinary differential equations 


$$
r^{\prime}=0, \quad r(0)=\rho
$$

and

$$
w^{\prime}+\lambda(t+1)^{-1} r^{2 / n}=0, \quad w(0)=0 .
$$

The explicit solution is as follows

$$
r(t)=\rho, \quad w(t)=-\lambda \rho^{2 / n} \log (t+1) .
$$

Thus the solution of (1.1) in the form (1.2) is represented as

$$
u(t, x)=\rho(i(t+1))^{-n / 2} e^{i|x|^{2} / 2(t+1)} \exp \left(-i \lambda \rho^{2 / n} \log (t+1)\right) .
$$

This solution does not belong to $\mathbf{L}^{2}$. However we have

$$
|u(t, x)|=\rho(t+1)^{-n / 2}
$$

and

$$
\|\mathcal{U}(-t-1) u(t)\|_{\dot{\mathbf{H}}^{\sigma}}=0 .
$$

Therefore our results contain the special solution (1.3).

\section{Local existence.}

In the next lemma we show the estimates of the remainder terms

$$
\begin{aligned}
& R_{1}=|\mathcal{V}(t+1) \varphi|^{2 / n} \mathcal{V}(t+1) \varphi-|\varphi|^{2 / n} \varphi, \\
& R_{2}=\left(\mathcal{V}^{*}(t+1)-1\right)|\mathcal{V}(t+1) \varphi|^{2 / n} \mathcal{V}(t+1) \varphi,
\end{aligned}
$$

where we denote $\varphi=\mathcal{F U}(-1-t) u$ and $\mathcal{V}(t+1)=\mathcal{F} M(t+1) \mathcal{F}^{-1}, \mathcal{V}^{*}(t+1)=\mathcal{V}(-t-1)$.

Lemma 2.1. Assume that

$$
\frac{2}{5} \rho \leq \inf _{\xi \in \mathbb{R}^{n}}|\varphi(t, \xi)| \leq\|\varphi(t)\|_{\mathbf{L}^{\infty}} \leq \frac{6}{5} \rho
$$

and $\|\varphi(t)\|_{\dot{\mathbf{H}}^{\sigma}} \leq \rho^{2}$ with $n / 2<\sigma<n / 2+2$. Then there exists a small $\rho>0$ such that the estimate

$$
\left\|R_{1}\right\|_{\mathbf{L}^{\infty}}+\left\|R_{2}\right\|_{\mathbf{L}^{\infty}} \leq C \rho^{2+2 / n}(t+1)^{-\delta}
$$

is true for all $t \geq 0$, where $\delta \in(0,(1 / 2)(\sigma-n / 2)$.

Proof. By the Sobolev embedding inequality

$$
\begin{aligned}
\|\phi\|_{\mathbf{L}^{\infty}} & \leq\|\widehat{\phi}\|_{\mathbf{L}^{1}} \leq C\left(\|\widehat{\phi}\|_{\dot{\mathbf{H}}^{0, n / 2-\nu}}+\|\widehat{\phi}\|_{\dot{\mathbf{H}}^{0, n / 2+\nu}}\right) \\
& =C\left(\|\phi\|_{\dot{\mathbf{H}}^{n / 2-\nu}}+\|\phi\|_{\dot{\mathbf{H}}^{n / 2+\nu}}\right)
\end{aligned}
$$


with small $\nu>0$. Also we have

$$
\begin{aligned}
& \|(\mathcal{V}(t+1)-1) \varphi\|_{\dot{\mathbf{H}}^{n / 2-\nu}} \\
& \quad=\left\|\mathcal{F}(M(t+1)-1) \mathcal{F}^{-1} \varphi\right\|_{\dot{\mathbf{H}}^{n / 2-\nu}} \\
& \quad \leq(t+1)^{-\sigma_{1} / 2}\left\|\mathcal{F}|\cdot|^{\sigma_{1}} \mathcal{F}^{-1} \varphi\right\|_{\dot{\mathbf{H}}^{n / 2-\nu}}=(t+1)^{-\sigma_{1} / 2}\|\varphi\|_{\dot{\mathbf{H}}^{n / 2-\nu+\sigma_{1}}}
\end{aligned}
$$

for $0 \leq \sigma_{1} \leq 2$. Similarly,

$$
\|(\mathcal{V}(t+1)-1) \varphi\|_{\dot{\mathbf{H}}^{n / 2+\nu}} \leq(t+1)^{-\sigma_{2} / 2}\|\varphi\|_{\dot{\mathbf{H}}^{n / 2+\nu+\sigma_{2}}}
$$

for $0 \leq \sigma_{2} \leq 2$. We take $n / 2+\nu+\sigma_{2}=n / 2-\nu+\sigma_{1}=\sigma$, then we find

$$
\begin{aligned}
\|(\mathcal{V}( & t+1)-1) \varphi \|_{\mathbf{L}^{\infty}} \\
& \leq C\|(\mathcal{V}(t+1)-1) \varphi\|_{\dot{\mathbf{H}}^{n / 2-\nu}}+C\|(\mathcal{V}(t+1)-1) \varphi\|_{\dot{\mathbf{H}}^{n / 2+\nu}} \\
& \leq C(t+1)^{-\delta}\|\varphi\|_{\dot{\mathbf{H}}^{\sigma}}
\end{aligned}
$$

for all $t \geq 0$, with $\delta \in(0,(1 / 2)(\sigma-n / 2))$, if $n / 2<\sigma<n / 2+2$. Also we write

$$
\begin{aligned}
\|\mathcal{V}(t+1) \varphi\|_{\mathbf{L}^{\infty}} & \leq\|\varphi\|_{\mathbf{L}^{\infty}}+\|(\mathcal{V}(t+1)-1) \varphi\|_{\mathbf{L}^{\infty}} \\
& \leq\|\varphi\|_{\mathbf{L}^{\infty}}+C(t+1)^{-\delta}\|\varphi\|_{\mathbf{H}^{\sigma}} .
\end{aligned}
$$

Hence the first term is estimated as

$$
\begin{aligned}
\left\|R_{1}\right\|_{\mathbf{L}^{\infty}} & \leq C\left(\|\mathcal{V}(t+1) \varphi\|_{\mathbf{L}^{\infty}}^{2 / n}+\|\varphi\|_{\mathbf{L}^{\infty}}^{2 / n}\right)\|(\mathcal{V}(t+1)-1) \varphi\|_{\mathbf{L}^{\infty}} \\
& \leq C(t+1)^{-\delta}\left(\|\varphi\|_{\mathbf{L}^{\infty}}+(t+1)^{-\delta}\|\varphi\|_{\dot{\mathbf{H}}^{\sigma}}\right)^{2 / n}\|\varphi\|_{\dot{\mathbf{H}}^{\sigma}} \\
& \leq C(t+1)^{-\delta} \rho^{2 / n+2}
\end{aligned}
$$

if $n / 2<\sigma<n / 2+2$.

Let us consider the estimate of $R_{2}$. In the same way as above we have

$$
\begin{aligned}
& \left\|\left(\mathcal{V}^{*}(t+1)-1\right)|\mathcal{V}(t+1) \varphi|^{2 / n} \mathcal{V}(t+1) \varphi\right\|_{\mathbf{L}^{\infty}} \\
& \quad \leq C(t+1)^{-\delta}\left\||\mathcal{V}(t+1) \varphi|^{2 / n} \mathcal{V}(t+1) \varphi\right\|_{\dot{\mathbf{H}}^{\sigma}},
\end{aligned}
$$

where $\delta \in(0,(1 / 2)(\sigma-n / 2)), n / 2<\sigma<n / 2+2$. By a generalized Leibniz rule (see Lemmas A1-A4 in the appendix of [11], also Lemma 2.2 in $[\mathbf{3}])$ we have

$$
\|u v\|_{\dot{\mathbf{H}}_{r}^{\sigma}} \leq C\|u\|_{\mathbf{L}^{q_{1}}}\|v\|_{\dot{\mathbf{H}}_{r_{1}}^{\sigma}}+C\|v\|_{\mathbf{L}^{q_{2}}}\|u\|_{\dot{\mathbf{H}}_{r_{2}}^{\sigma}}
$$

for $\sigma \geq 0,1 / r=1 / q_{1}+1 / r_{1}=1 / q_{2}+1 / r_{2}, 1<q_{1}, q_{2} \leq \infty, 1<r_{1}, r_{2}<\infty$. Hence taking $q_{1}=q_{2}=\infty, r_{1}=r_{2}=2$ we find

$$
\begin{aligned}
\left\||u|^{2 / n} u\right\|_{\dot{\mathbf{H}}^{\sigma}} & =\left\|u^{1 / n} \bar{u}^{1 / n} u\right\|_{\dot{\mathbf{H}}^{\sigma}} \\
& \leq C\|u\|_{\mathbf{L}^{\infty}}^{2 / n}\|u\|_{\dot{\mathbf{H}}^{\sigma}}+C\|u\|_{\mathbf{L}^{\infty}}^{1+1 / n}\left(\left\|u^{1 / n}\right\|_{\dot{\mathbf{H}}^{\sigma}}+\left\|\bar{u}^{1 / n}\right\|_{\dot{\mathbf{H}}^{\sigma}}\right) .
\end{aligned}
$$

Let $\sigma=m+\nu, m \in \mathbb{N}, \nu \in[0,1)$. We let $\mu=1 / n$. By the Leibniz rule we find 


$$
\left\|\partial_{x_{j}}^{m} u^{\mu}\right\|_{\dot{\mathbf{H}}^{\nu}} \leq C \sum_{k=1}^{m}\left\|u^{\mu-k} \partial_{x_{j}}^{m-k}\left(\partial_{x_{j}} u\right)^{k}\right\|_{\dot{\mathbf{H}}^{\nu}}
$$

with $\mu=1 / n$. We use the Gagliardo-Nirenberg interpolation inequality (see Theorem 2.44 in $[\mathbf{1}])$

$$
\|u\|_{\dot{\mathbf{H}}_{p}^{\alpha}} \leq C\|u\|_{\mathbf{L}^{q}}^{1-\alpha / s}\|u\|_{\dot{\mathbf{H}}_{r}^{s}}^{\alpha / s}
$$

for $1<q, r \leq \infty, 0<\alpha<s, s / p=(s-\alpha) / q+\alpha / r$. In particular, choosing $q=\infty$, $r=2 \sigma, s=1$, we find

$$
\|u\|_{\dot{\mathbf{H}}_{2 \sigma / \nu}^{\nu}} \leq C\|u\|_{\mathbf{L}^{\infty}}^{1-\nu}\|u\|_{\dot{\mathbf{H}}_{2 \sigma}^{1}}^{\nu}
$$

and taking $q=\infty, r=2, s=\sigma$, we have

$$
\|u\|_{\dot{\mathbf{H}}_{2 \sigma / \alpha}^{\alpha}} \leq C\|u\|_{\mathbf{L}^{\infty}}^{1-\alpha / \sigma}\|u\|_{\dot{\mathbf{H}}^{\sigma}}^{\alpha / \sigma}
$$

for $0<\alpha<\sigma$. Therefore we get

$$
\begin{aligned}
\left\|u^{\mu-k}\right\|_{\dot{\mathbf{H}}_{2 \sigma / \nu}^{\nu}} & \leq C\left\|u^{\mu-k}\right\|_{\mathbf{L}^{\infty}}^{1-\nu}\left\|u^{\mu-k}\right\|_{\dot{\mathbf{H}}_{2 \sigma}^{1}}^{\nu} \\
& \leq C\left\|u^{\mu-k}\right\|_{\mathbf{L}^{\infty}}^{1-\nu}\left\|u^{\mu-k-1}\right\|_{\mathbf{L}^{\infty}}^{\nu}\|u\|_{\dot{\mathbf{H}}_{2 \sigma}^{1}}^{\nu} \\
& \leq C\left\|u^{\mu-k}\right\|_{\mathbf{L}^{\infty}}^{1-\nu}\left\|u^{\mu-k-1}\right\|_{\mathbf{L}^{\infty}}^{\nu}\|u\|_{\mathbf{L}^{\infty}}^{\nu-\nu / \sigma}\|u\|_{\dot{\mathbf{H}}^{\sigma}}^{\nu / \sigma} .
\end{aligned}
$$

Hence

$$
\begin{aligned}
& \left\|u^{\mu-k} \partial_{x_{j}}^{m-k}\left(\partial_{x_{j}} u\right)^{k}\right\|_{\dot{\mathbf{H}}^{\nu}} \\
& \quad \leq C\left\|u^{\mu-k}\right\|_{\mathbf{L}^{\infty}}\|u\|_{\dot{\mathbf{H}}_{2 \sigma}^{1}}^{k-1}\|u\|_{\dot{\mathbf{H}}_{2 \sigma /(\sigma+1-k)}^{\sigma+1-k}}+C\left\|u^{\mu-k}\right\|_{\dot{\mathbf{H}}_{2 \sigma / \nu}^{\nu}}\|u\|_{\dot{\mathbf{H}}_{2 \sigma}^{1}}^{k-1}\|u\|_{\dot{\mathbf{H}}_{2 \sigma /(m+1-k)}^{m+1-k}} \\
& \quad \leq C\left\|u^{\mu-k}\right\|_{\mathbf{L}^{\infty}}\|u\|_{\mathbf{L}^{\infty}}^{k-1}\|u\|_{\dot{\mathbf{H}}^{\sigma}}+C\left\|u^{\mu-k}\right\|_{\mathbf{L}^{\infty}}^{1-\nu}\left\|u^{\mu-k-1}\right\|_{\mathbf{L}^{\infty}}^{\nu}\|u\|_{\mathbf{L}^{\infty}}^{k-1+\nu}\|u\|_{\dot{\mathbf{H}}^{\sigma}} .
\end{aligned}
$$

Thus we obtain

$$
\left\||u|^{2 / n} u\right\|_{\dot{\mathbf{H}}^{\sigma}} \leq C\|u\|_{\mathbf{L}^{\infty}}^{2 / n}\|u\|_{\dot{\mathbf{H}}^{\sigma}}+C \sum_{k=1}^{m}\|u\|_{\mathbf{L}^{\infty}}^{2 / n+k+1}\left(\inf _{x \in \mathbb{R}^{n}}|u|\right)^{-k-1}\|u\|_{\dot{\mathbf{H}}^{\sigma}} .
$$

We apply the above estimate with $u=\mathcal{V}(t+1) \varphi$ to get for $n \geq 2$

$$
\begin{aligned}
&\left\||\mathcal{V}(t+1) \varphi|^{2 / n} \mathcal{V}(t+1) \varphi\right\|_{\dot{\mathbf{H}}^{\sigma}} \\
& \leq C\|\mathcal{V}(t+1) \varphi\|_{\mathbf{L}^{\infty}}^{2 / n}\|\mathcal{V}(t+1) \varphi\|_{\dot{\mathbf{H}}^{\sigma}} \\
&+C \sum_{k=1}^{m}\|\mathcal{V}(t+1) \varphi\|_{\mathbf{L}^{\infty}}^{2 / n+k+1}\left(\inf _{x \in \mathbb{R}^{n}}|\mathcal{V}(t+1) \varphi|\right)^{-k-1}\|\mathcal{V}(t+1) \varphi\|_{\dot{\mathbf{H}}^{\sigma}} .
\end{aligned}
$$

Our assumption says that

$$
\inf _{x \in \mathbb{R}^{n}}|\mathcal{V}(t+1) \varphi| \geq \inf _{\xi \in \mathbb{R}^{n}}|\varphi|-C(t+1)^{(-1 / 2)(\sigma-n / 2)}\|\mathcal{V}(t+1) \varphi\|_{\dot{\mathbf{H}}^{\sigma}}
$$




$$
\geq \frac{2}{5} \rho-C(t+1)^{(-1 / 2)(\sigma-n / 2)} \rho^{2} \geq \frac{1}{3} \rho .
$$

Therefore by $(2.2)$

$$
\begin{aligned}
& \left\||\mathcal{V}(t+1) \varphi|^{2 / n} \mathcal{V}(t+1) \varphi\right\|_{\dot{\mathbf{H}}^{\sigma}} \\
& \quad \leq C \rho^{2 / n}\|\mathcal{V}(t+1) \varphi\|_{\dot{\mathbf{H}}^{\sigma}}=C \rho^{2 / n}\|\varphi\|_{\dot{\mathbf{H}}^{\sigma}} \leq C \rho^{2 / n+2} .
\end{aligned}
$$

Substitution of (2.4) into (2.3) yields

$$
\left\|R_{2}\right\|_{\mathbf{L}^{\infty}} \leq C \rho^{2 / n+2}(t+1)^{-\delta} .
$$

Lemma 2.1 is proved.

To prove local existence we introduce the function space $\mathbf{X}_{T}$ such that

$$
\mathbf{X}_{T}=\left\{v \in \mathbf{C}\left([0, T] ; \mathbf{L}^{\infty} \cap \mathbf{C}\right) ;\|v\|_{\mathbf{X}_{T}}<\infty\right\},
$$

where

$$
\|v\|_{\mathbf{X}_{T}}=\sup _{0 \leq t \leq T}\left(\|\mathcal{F} \mathcal{U}(-t-1) v(t)\|_{\mathbf{L}^{\infty}}+\|\mathcal{F} \mathcal{U}(-t-1) v(t)\|_{\dot{\mathbf{H}}^{\sigma}}\right)
$$

with $n / 2<\sigma<n / 2+2$. We are now in a position to prove the local existence theorem.

LEMma 2.2. Assume that the initial data satisfy

$$
\frac{\rho}{2} \leq \inf _{\xi \in \mathbb{R}^{n}}\left|\widehat{u_{0}}(\xi)\right| \leq\left\|\widehat{u_{0}}\right\|_{\mathbf{L}^{\infty}} \leq \rho
$$

and $\left\|e^{i(1 / 2)|\xi|^{2}} \widehat{u_{0}}\right\|_{\dot{\mathbf{H}}^{\sigma}} \leq \rho^{2}$ with $n / 2<\sigma<n / 2+2$. Then there exist a time $T$ such that the Cauchy problem (1.1) has a unique solution in $\mathbf{X}_{T}$ satisfying the estimates

$$
\frac{2}{5} \rho \leq \inf _{0 \leq t \leq T} \inf _{\xi \in \mathbb{R}^{n}}|(\mathcal{F U}(-t-1) u)(t, \xi)| \leq \sup _{0 \leq t \leq T}\|\mathcal{F U}(-t-1) u\|_{\mathbf{L}^{\infty}} \leq \frac{6}{5} \rho .
$$

ProOF. Let us consider the linearized problem corresponding to the Cauchy problem (1.1)

$$
i \partial_{t} u+\frac{1}{2} \Delta u=\lambda|v|^{2 / n} v, \quad u(0, x)=u_{0}(x)
$$

where $v \in \mathbf{X}_{T}$,

$$
\sup _{0 \leq t \leq T}\|\mathcal{F U}(-t-1) v(t)\|_{\mathbf{L}^{\infty}} \leq \frac{6}{5} \rho, \quad \sup _{0 \leq t \leq T}\|\mathcal{F} \mathcal{U}(-t-1) v(t)\|_{\dot{\mathbf{H}}^{\sigma}} \leq \frac{6}{5} \rho^{2} .
$$

Consider the integral equation associated with (2.5)

$$
\varphi(t)=\varphi(0)-i \lambda \int_{0}^{t} \mathcal{F U}(-\tau-1) F\left(\mathcal{U}(\tau+1) \mathcal{F}^{-1} \widetilde{\varphi}(\tau)\right) d \tau,
$$


where

$$
F(\phi)=|\phi|^{2 / n} \phi, \quad \varphi(t) \equiv \mathcal{F U}(-t-1) u(t), \quad \widetilde{\varphi}(t) \equiv \mathcal{F U}(-t-1) v(t) .
$$

Then using the factorization formulas

$$
\begin{aligned}
& \mathcal{U}(t+1) \mathcal{F}^{-1}=M(t+1) D_{t+1} \mathcal{V}(t+1), \\
& \mathcal{F U}(-t-1)=\mathcal{V}^{*}(t+1) D_{t+1}^{-1} \bar{M}(t+1),
\end{aligned}
$$

with $\mathcal{V}(t+1)=\mathcal{F} M(t+1) \mathcal{F}^{-1}$ and $\mathcal{V}^{*}(t+1)=\mathcal{V}(-t-1)$ we get

$$
\varphi(t)=\varphi(0)-i \lambda \int_{0}^{t}(\tau+1)^{-1} \mathcal{V}^{*}(\tau+1) F(\mathcal{V}(\tau+1) \widetilde{\varphi}(\tau)) d \tau
$$

As in the estimate (2.4), we obtain

$$
\|\varphi(t)\|_{\dot{\mathbf{H}}^{\sigma}} \leq\left\|e^{(i / 2)|\xi|^{2}} \widehat{u_{0}}\right\|_{\dot{\mathbf{H}}^{\sigma}}+C \rho^{2 / n+2} \int_{0}^{t}(\tau+1)^{-1} d \tau,
$$

since $\varphi(0)=\mathcal{F U}(-1) u_{0}=e^{(i / 2)|\xi|^{2}} \widehat{u_{0}}(\xi)$. Hence we find there exists a time $T=T(\rho)>0$ such that

$$
\begin{aligned}
\sup _{0 \leq t \leq T}\|\varphi(t)\|_{\dot{\mathbf{H}}^{\sigma}} & \leq\left\|e^{(i / 2)|\xi|^{2}} \widehat{u_{0}}\right\|_{\dot{\mathbf{H}}^{\sigma}}+C \rho^{2 / n+2} \log (T+1) \\
& \leq \rho^{2}+C \rho^{2 / n+2} \log (T+1) \leq \frac{6}{5} \rho^{2} .
\end{aligned}
$$

We also have by Lemma 2.1

$$
\begin{aligned}
|\varphi(t, \xi)| & \leq \rho+C \rho^{2 / n+1} \int_{0}^{t}(\tau+1)^{-1} d \tau+C \rho^{2 / n+2} \int_{0}^{t}(\tau+1)^{-1-(1 / 2)(\sigma-n / 2)} d \tau \\
& \leq \rho+C \rho^{2 / n+1} \log (T+1)+C \rho^{2 / n+2}
\end{aligned}
$$

and

$$
\begin{aligned}
|\varphi(t, \xi)| & \geq \frac{1}{2} \rho-C \rho^{2 / n+1} \int_{0}^{t}(\tau+1)^{-1} d \tau-C \rho^{2 / n+2} \int_{0}^{t}(\tau+1)^{-1-(1 / 2)(\sigma-n / 2)} d \tau \\
& \geq \frac{1}{2} \rho-C \rho^{2 / n+1} \log (T+1)-C \rho^{2 / n+2}
\end{aligned}
$$

Hence, there exists a time $T=T(\rho)>0$ such that

$$
\sup _{0 \leq t \leq T}\|\varphi(t)\|_{\mathbf{L}^{\infty}} \leq \rho+C \rho^{2 / n+1} \log (T+1) \leq \frac{6}{5} \rho .
$$

Therefore the mapping $S$ defined by $u=S v$ transforms $\mathbf{X}_{T}$ into itself. Also the lower bound

$$
\inf _{0 \leq t \leq T} \inf _{\xi \in \mathbb{R}^{n}}|\varphi(t, \xi)| \geq \frac{2}{5} \rho
$$


is true. Let $\varphi_{1}$ and $\varphi_{2}$ be solutions to the integral equations

$$
\varphi_{j}(t)=e^{(i / 2)|\xi|^{2}} \widehat{u_{0}}-i \lambda \int_{0}^{t}(\tau+1)^{-1} \mathcal{V}^{*}(\tau+1) F\left(\mathcal{V}(\tau+1) \widetilde{\varphi}_{j}(\tau)\right) d \tau
$$

Then

$$
\begin{aligned}
\varphi_{1}(t) & -\varphi_{2}(t) \\
& =-i \lambda \int_{0}^{t}(\tau+1)^{(-n / 2)(2 / n)} \mathcal{V}^{*}(\tau+1)\left(F\left(\mathcal{V}(\tau+1) \widetilde{\varphi}_{1}(\tau)\right)-F\left(\mathcal{V}(\tau+1) \widetilde{\varphi}_{2}(\tau)\right)\right) d \tau .
\end{aligned}
$$

Therefore as above, by Lemma 2.1, we find that

$$
\begin{aligned}
& \left\|\varphi_{1}(t)-\varphi_{2}(t)\right\|_{\dot{\mathbf{H}}^{\sigma}}+\left\|\varphi_{1}(t)-\varphi_{2}(t)\right\|_{\mathbf{L}^{\infty}} \\
& \quad \leq C \rho^{2 / n} \log (T+1)\left(\left\|\widetilde{\varphi}_{1}(t)-\widetilde{\varphi}_{2}(t)\right\|_{\dot{\mathbf{H}}^{\sigma}}+\left\|\widetilde{\varphi}_{1}(t)-\widetilde{\varphi}_{2}(t)\right\|_{\mathbf{L}^{\infty}}\right)
\end{aligned}
$$

which implies there exists a time $T$ such that $S$ is a contraction mapping in $\mathbf{X}_{T}$. Lemma 2.2 is proved.

\section{Proof of Theorem 1.1.}

We prove Theorem 1.1 by showing a-priori estimates of the local solutions obtained in Lemma 2.2. We now state our results.

Lemma 3.1. Assume that the assumptions of Theorem 1.1 hold. Also suppose that

$$
\sup _{t \in[0, T]}(t+1)^{-\gamma}\|\varphi(t)\|_{\dot{\mathbf{H}}^{\sigma}} \leq \frac{6}{5} \rho^{2}
$$

for some $T>0$, where $\gamma$ is small positive number satisfying $5 \rho^{2 / n}<\gamma<(1 / n)(\sigma-n / 2)$. Then the estimate

$$
\frac{2}{5} \rho<\inf _{t \in[0, T]} \inf _{\xi \in \mathbb{R}^{n}}|\varphi(t, \xi)| \leq \sup _{t \in[0, T]}\|\varphi(t)\|_{\mathbf{L}^{\infty}}<\frac{6}{5} \rho
$$

is true for sufficiently small $\rho>0$, where $\varphi(t)=\mathcal{F U}(-t-1) u(t)$.

Proof. By the contrary we may assume that there exists a first time $T>0$ such that

$$
\inf _{t \in[0, T]} \inf _{\xi \in \mathbb{R}^{n}}|\varphi(t, \xi)|=\frac{2}{5} \rho
$$

or

$$
\sup _{t \in[0, T]}\|\varphi(t)\|_{\mathbf{L}^{\infty}}=\frac{6}{5} \rho .
$$

We represent the solution of (1.1) in the form $\varphi(t)=\mathcal{F U}(-t-1) u(t)=r e^{i w}, r=|\varphi|$, $w=\arg \varphi$, then applying the factorization formulas $\mathcal{U}(t+1) \mathcal{F}^{-1}=M(t+1) D_{t+1} \mathcal{V}(t+1)$, 
$\mathcal{F U}(-t-1)=\mathcal{V}^{*}(t+1) D_{t+1}^{-1} \bar{M}(t+1)$, with $\mathcal{V}(t+1)=\mathcal{F} M(t+1) \mathcal{F}^{-1}$ and $\mathcal{V}^{*}(t+1)=$ $\mathcal{V}(-t-1)$, we find

$$
\left\{\begin{array}{l}
\partial_{t} r=g_{1}(t), \\
\partial_{t} w=-\lambda(t+1)^{-1} r^{2 / n}-g_{2}(t),
\end{array}\right.
$$

where

$$
\begin{aligned}
& g_{1}(t)=\lambda(t+1)^{-1} \operatorname{Im}\left(e^{-i w}\left(R_{1}+R_{2}\right)\right), \\
& g_{2}(t)=r^{-1} \lambda(t+1)^{-1} \operatorname{Re}\left(e^{-i w}\left(R_{1}+R_{2}\right)\right)
\end{aligned}
$$

with the remainder terms

$$
\begin{aligned}
& R_{1}=|\mathcal{V}(t+1) \varphi|^{2 / n} \mathcal{V}(t+1) \varphi-|\varphi|^{2 / n} \varphi \\
& R_{2}=\left(\mathcal{V}^{*}(t+1)-1\right)|\mathcal{V}(t+1) \varphi|^{2 / n} \mathcal{V}(t+1) \varphi
\end{aligned}
$$

By Lemma 2.1, we have

$$
\left\|g_{1}(t)\right\|_{\mathbf{L}^{\infty}} \leq C \rho^{2 / n+2}(t+1)^{\gamma-\delta-1},
$$

where $\delta \in(0,(1 / 2)(\sigma-n / 2))$. Hence integrating equation $\partial_{t} r=g_{1}(t)$ we find

$$
\begin{aligned}
\inf _{t \in[0, T]} \inf _{\xi \in \mathbb{R}^{n}}|\varphi(t, \xi)| & =\inf _{t \in[0, T]} \inf _{\xi \in \mathbb{R}^{n}} r(t) \\
& \geq \inf _{\xi \in \mathbb{R}^{n}}\left|\widehat{u_{0}}(\xi)\right|-C \rho^{2 / n+2}(t+1)^{\gamma-\delta} \geq \frac{1}{2} \rho>\frac{2}{5} \rho
\end{aligned}
$$

and

$$
\sup _{t \in[0, T]}\|\varphi(t)\|_{\mathbf{L}^{\infty}} \leq \frac{11}{10} \rho<\frac{6}{5} \rho .
$$

This is a desired contradiction and the lemma is proved.

Lemma 3.2. Assume that the assumptions of Theorem 1.1 are true. Let the estimates

$$
\frac{2}{5} \rho \leq \inf _{t \in[0, T]} \inf _{\xi \in \mathbb{R}^{n}}|\varphi(t, \xi)| \leq \sup _{t \in[0, T]}\|\varphi(t)\|_{\mathbf{L}^{\infty}} \leq \frac{6}{5} \rho
$$

hold for some $T>0$. Then the estimate

$$
\sup _{t \in[0, T]}(t+1)^{-\gamma}\|\varphi(t)\|_{\dot{\mathbf{H}}^{\sigma}}<\frac{6}{5} \rho^{2}
$$

is valid for sufficiently small $\rho>0$ and $5 \rho^{2 / n}<\gamma$, where $\varphi(t)=\mathcal{F U}(-t-1) u(t)$.

Proof. By the contrary we assume that there exists the first time $T$ such that 


$$
\sup _{t \in[0, T]}(t+1)^{-\gamma}\|\varphi(t)\|_{\dot{\mathbf{H}}^{\sigma}}=\frac{6}{5} \rho^{2}
$$

We now turn to the integral equation

$$
\varphi(t)=\varphi(0)-i \lambda \int_{0}^{t}(\tau+1)^{-1} \mathcal{V}^{*}(\tau+1) F(\mathcal{V}(\tau+1) \varphi(\tau)) d \tau
$$

As in estimate (2.4) we get

$$
\begin{aligned}
\|\varphi(t)\|_{\dot{\mathbf{H}}^{\sigma}} & \leq\|\varphi(0)\|_{\dot{\mathbf{H}}^{\sigma}}+C \rho^{2 / n} \int_{0}^{t}(\tau+1)^{-1}\|\varphi(\tau)\|_{\dot{\mathbf{H}}^{\sigma}} d \tau \\
& \leq\|\varphi(0)\|_{\dot{\mathbf{H}}^{\sigma}}+C \rho^{2 / n+2} \int_{0}^{t}(\tau+1)^{\gamma-1} d \tau \\
& \leq \rho^{2}\left(1+\rho^{2 / n} \frac{1}{\gamma}(t+1)^{\gamma}\right)<\frac{6}{5} \rho^{2}(t+1)^{\gamma},
\end{aligned}
$$

if $5 \rho^{2 / n}<\gamma$. This is a desired contradiction, which completes the proof of the lemma.

Proof of Theorem 1.1. By Lemmas 3.1 and 3.2 we have a priori estimates of solutions in the space $\mathbf{X}_{T}$. Therefore the global in time existence of small solutions follows. The desired time decay of solutions can be obtained by factorization technique

$$
\begin{aligned}
\|u(t)\|_{\mathbf{L}^{\infty}} & =\left\|D_{t+1} \mathcal{V}(t+1) \varphi\right\|_{\mathbf{L}^{\infty}} \\
& \leq(t+1)^{-n / 2}\|\varphi(\tau)\|_{\mathbf{L}^{\infty}}+C(t+1)^{-n / 2-\delta}\|\varphi\|_{\dot{\mathbf{H}}^{\sigma}} \\
& \leq \frac{6}{5} \rho(t+1)^{-n / 2}+C \rho^{2}(t+1)^{\gamma-n / 2-\delta} \leq \frac{7}{5} \rho(t+1)^{-n / 2}
\end{aligned}
$$

and

$$
\begin{aligned}
\inf _{x \in \mathbb{R}^{n}}|u(t)| & =\inf _{x \in \mathbb{R}^{n}}\left|D_{t+1} \mathcal{V}(t+1) \varphi\right| \\
& \geq(t+1)^{-n / 2} \inf _{x \in \mathbb{R}^{n}}\left|\varphi\left(\frac{x}{t+1}, t\right)\right|-C(t+1)^{-n / 2-\delta}\|\varphi\|_{\dot{\mathbf{H}}^{\sigma}} \\
& \geq \frac{2}{5} \rho(t+1)^{-n / 2}-C \rho^{2}(t+1)^{\gamma-n / 2-\delta} \geq \frac{1}{5} \rho(t+1)^{-n / 2}
\end{aligned}
$$

where $\delta \in(0,(1 / 2)(\sigma-n / 2))$.

\section{Proof of Theorem 1.2.}

We have the existence of the final states in Theorem 1.2 by the lemma below. Denote

$$
y(t)=e^{i \lambda \int_{0}^{t}(\tau+1)^{-1}|\varphi(\tau)|^{2 / n} d \tau} \varphi(t) .
$$

LEMma 4.1. Let the initial data satisfy the assumptions of Theorem 1.1 and $u$ be the solution constructed in Theorem 1.1. Then there exists a unique final state $y_{+} \in \mathbf{L}^{\infty} \cap \dot{\mathbf{H}}^{\beta}$ with $n / 2<\beta<\sigma-2 \gamma, 5 \rho^{2 / n}<\gamma<(1 / n)(\sigma-n / 2)$ such that 


$$
\begin{aligned}
& \left\|y(t)-y_{+}\right\|_{\mathbf{L}^{\infty}} \leq C \rho^{2 / n+2}(t+1)^{-\delta+\gamma} \\
& \left\|y(t)-y_{+}\right\|_{\dot{\mathbf{H}}^{\beta}} \leq C \rho^{2 / n+2}(t+1)^{(-1 / 2)(\sigma-\beta)+\gamma}
\end{aligned}
$$

for all $t>0$, where $\delta \in(0,(1 / 2)(\sigma-n / 2))$ and

$$
\frac{1}{5} \rho \leq \inf _{\xi \in \mathbb{R}^{n}}\left|y_{+}(\xi)\right| \leq\left\|y_{+}\right\|_{\mathbf{L}^{\infty}} \leq \frac{7}{5} \rho .
$$

Proof. Multiplying both sides of $(1.1)$ by $\mathcal{F U}(-t-1)$ via the factorization formulas we obtain

$$
i \partial_{t} \varphi(t)=\lambda(t+1)^{-1}|\varphi(t)|^{2 / n} \varphi(t)+\lambda(t+1)^{-1}\left(R_{1}+R_{2}\right),
$$

where $\varphi(t)=\mathcal{F U}(-t-1) u$ and the remainder terms

$$
\begin{aligned}
& R_{1}=|\mathcal{V}(t+1) \varphi|^{2 / n} \mathcal{V}(t+1) \varphi-|\varphi|^{2 / n} \varphi, \\
& R_{2}=\left(\mathcal{V}^{*}(t+1)-1\right)|\mathcal{V}(t+1) \varphi|^{2 / n} \mathcal{V}(t+1) \varphi
\end{aligned}
$$

We multiply both sides of (4.1) by $e^{i \lambda \int_{0}^{t}(\tau+1)^{-1}|\varphi(\tau)|^{2 / n} d \tau}$

$$
i \partial_{t} y=\lambda e^{i \lambda \int_{0}^{t}(\tau+1)^{-1}|\varphi|^{2 / n} d \tau}(t+1)^{-1}\left(R_{1}+R_{2}\right)
$$

from which it follows that

$$
|y(t, \xi)-y(s, \xi)| \leq|\lambda| \int_{s}^{t}(\tau+1)^{-1}\left|R_{1}+R_{2}\right| d \tau .
$$

By Lemmas 3.1 and 3.2 we have

$$
\|\varphi(t)\|_{\mathbf{L}^{\infty}} \leq \frac{6}{5} \rho, \quad\|\varphi(t)\|_{\dot{\mathbf{H}}^{\sigma}} \leq \frac{6}{5} \rho^{2}(t+1)^{\gamma}
$$

for all $t>0$, where $5 \rho^{2 / n}<\gamma<(1 / n)(\sigma-n / 2)$. As in the proof of Lemma 2.1, we get

$$
\begin{aligned}
\|y(t)-y(s)\|_{\mathbf{L}^{\infty}} & \leq C \int_{s}^{t}(\tau+1)^{-1}\left|R_{1}+R_{2}\right| d \tau \\
& \leq C \rho^{2 / n+2} \int_{s}^{t}(\tau+1)^{-1-\delta+\gamma} d \tau \leq C \rho^{2 / n+2}(s+1)^{-\delta+\gamma}
\end{aligned}
$$

and

$$
\begin{aligned}
\| y(t) & -y(s) \|_{\dot{\mathbf{H}}^{\beta}} \\
& \leq C \int_{s}^{t}(\tau+1)^{-1}\left\|R_{1}+R_{2}\right\|_{\dot{\mathbf{H}}^{\beta}} d \tau \\
& \leq C \rho^{2 / n+2} \int_{s}^{t}(\tau+1)^{-1-(1 / 2)(\sigma-\beta)+\gamma} d \tau \leq C \rho^{2 / n+2}(s+1)^{(-1 / 2)(\sigma-\beta)+\gamma}
\end{aligned}
$$

for all $t \geq s>0$. Hence there exists a unique limit $y_{+} \in \mathbf{L}^{\infty} \cap \dot{\mathbf{H}}^{\beta}$ such that 


$$
\left\|y(t)-y_{+}\right\|_{\mathbf{L}^{\infty}} \leq C \rho^{2 / n+2}(t+1)^{-\delta+\gamma}
$$

and

$$
\left\|y(t)-y_{+}\right\|_{\dot{\mathbf{H}}^{\beta}} \leq C \rho^{2 / n+2}(t+1)^{(-1 / 2)(\sigma-\beta)+\gamma}
$$

for all $t \geq 0$. Since $(2 / 5) \rho \leq|y(0, \xi)| \leq(6 / 5) \rho$ and

$$
|y(0, \xi)|+\left|y_{+}(\xi)-y(0, \xi)\right| \geq\left|y_{+}(\xi)\right| \geq|y(0, \xi)|-\left|y_{+}(\xi)-y(0, \xi)\right|,
$$

we have the estimates

$$
\begin{aligned}
\frac{1}{5} \rho & \leq \frac{2}{5} \rho-\left\|y_{+}-y(0)\right\|_{\mathbf{L}^{\infty}} \\
& \leq \inf _{\xi \in \mathbb{R}^{n}}\left|y_{+}(\xi)\right| \\
& \leq\left\|y_{+}\right\|_{\mathbf{L}^{\infty}} \leq \frac{6}{5} \rho+\left\|y_{+}-y(0)\right\|_{\mathbf{L}^{\infty}} \leq \frac{7}{5} \rho .
\end{aligned}
$$

This completes of the proof of the lemma.

The asymptotics of solutions in Theorem 1.2 follows from the lemma below.

LEMma 4.2. Let $u$ be the solution constructed in Theorem 1.1. Then there exists a unique final state $\widehat{u_{+}} \in \mathbf{L}^{\infty} \cap \dot{\mathbf{H}}^{\beta}$ with $n / 2<\beta<\sigma<n / 2+2$ such that the following estimates

$$
\begin{aligned}
\| u(t) & -M(t+1) D_{t+1} \widehat{u_{+}} e^{-i \lambda\left|\widehat{u_{+}}\right|^{2 / n} \log (t+1)} \|_{\mathbf{L}^{\infty}} \\
& \leq C \rho^{2}(t+1)^{-n / 2-\delta+\gamma}+C \rho^{(2 / n+2)(2 / n)+1}(t+1)^{-n / 2-(2 / n)(\delta-\gamma)} \log (t+1)
\end{aligned}
$$

and

$$
\begin{aligned}
& \left\|\mathcal{F} \mathcal{U}(-t-1) u(t)-\widehat{u_{+}} e^{-i \lambda\left|\widehat{u_{+}}\right|^{2 / n} \log (t+1)}\right\|_{\dot{\mathbf{H}}^{\beta}} \\
& \quad \leq C \rho^{4 / n+2}(t+1)^{(-1 / 2)(\sigma-\beta)+\gamma} \log (t+1)
\end{aligned}
$$

hold for all $t>0$. Furthermore we have

$$
\frac{1}{5} \rho \leq \inf _{\xi \in \mathbb{R}^{n}}\left|\widehat{u_{+}}(\xi)\right| \leq\left\|\widehat{u_{+}}\right\|_{\mathbf{L}^{\infty}} \leq \frac{7}{5} \rho,
$$

where $\delta \in(0,(1 / 2)(\sigma-n / 2)), 0<\gamma<(\sigma-\beta) / n$.

Proof. We consider the asymptotics of

$$
\varphi(t)=\mathcal{F U}(-t-1) u(t)=e^{-i \lambda \int_{0}^{t}(\tau+1)^{-1}|\varphi(\tau)|^{2 / n} d \tau} y(t) .
$$

We put

$$
\Psi(t)=\int_{0}^{t}(\tau+1)^{-1}\left(|\varphi(\tau)|^{2 / n}-|\varphi(t)|^{2 / n}\right) d \tau
$$


then

$$
\begin{aligned}
\Psi(t)-\Psi(s)= & \int_{s}^{t}(\tau+1)^{-1}\left(|\varphi(\tau)|^{2 / n}-|\varphi(t)|^{2 / n}\right) d \tau \\
& -\left(|\varphi(t)|^{2 / n}-|\varphi(s)|^{2 / n}\right) \log (s+1)
\end{aligned}
$$

for $0<s<\tau<t$. Hence by Lemma 4.1

$$
\begin{aligned}
\| \Psi(t) & -\Psi(s) \|_{\mathbf{L}^{\infty}} \\
\leq & C \int_{s}^{t}(\tau+1)^{-1}\|y(\tau)-y(t)\|_{\mathbf{L}^{\infty}}^{2 / n} d \tau+C\|y(t)-y(s)\|_{\mathbf{L}^{\infty}}^{2 / n} \log (s+1) \\
\leq & C \rho^{(2 / n+2)(2 / n)} \int_{s}^{t}(\tau+1)^{-1-(2 / n)(\delta-\gamma)} d \tau \\
& +C \rho^{(2 / n+2)(2 / n)}(s+1)^{(-2 / n)(\delta-\gamma)} \log (s+1) \\
\leq & C \rho^{(2 / n+2)(2 / n)}(s+1)^{(-2 / n)(\delta-\gamma)} \log (s+1)
\end{aligned}
$$

and in the same way as in the proof of Lemma 2.1, we have by Lemma 4.1

$$
\begin{aligned}
\|\Psi(t)-\Psi(s)\|_{\dot{\mathbf{H}}^{\beta}} \leq & C \int_{s}^{t}(\tau+1)^{-1}\left\||y(\tau)|^{2 / n}-|y(t)|^{2 / n}\right\|_{\dot{\mathbf{H}}^{\beta}} d \tau \\
& +C\left\||y(\tau)|^{2 / n}-|y(t)|^{2 / n}\right\|_{\dot{\mathbf{H}}^{\beta}} \log (s+1) \\
\leq & C \rho^{2 / n-1} \int_{s}^{t}(\tau+1)^{-1}\|y(\tau)-y(t)\|_{\dot{\mathbf{H}}^{\beta}} d \tau \\
& +C \rho^{2 / n-1}\|y(\tau)-y(t)\|_{\dot{\mathbf{H}}^{\beta}} \log (s+1) \\
\leq & C \rho^{4 / n+1} \int_{s}^{t}(\tau+1)^{-1-(1 / 2)(\sigma-\beta)+\gamma} d \tau \\
& +C \rho^{4 / n+1}(s+1)^{(-1 / 2)(\sigma-\beta)+\gamma} \log (s+1) \\
\leq & C \rho^{4 / n+1}(s+1)^{(-1 / 2)(\sigma-\beta)+\gamma} \log (s+1) .
\end{aligned}
$$

Therefore there exists a unique $\Psi_{+} \in \mathbf{L}^{\infty} \cap \dot{\mathbf{H}}^{\beta}$ such that

$$
\left\|\Psi(t)-\Psi_{+}\right\|_{\mathbf{L}^{\infty}} \leq C \rho^{(2 / n+2)(2 / n)}(t+1)^{(-2 / n)(\delta-\gamma)} \log (t+1)
$$

and

$$
\left\|\Psi(t)-\Psi_{+}\right\|_{\dot{\mathbf{H}}^{\beta}} \leq C \rho^{4 / n+1}(t+1)^{(-1 / 2)(\sigma-\beta)+\gamma} \log (t+1) .
$$

Since

$$
\Psi(t)=\int_{0}^{t}(\tau+1)^{-1}|\varphi(\tau)|^{2 / n} d \tau-|\varphi(t)|^{2 / n} \log (t+1)
$$

we have 


$$
\begin{gathered}
\left\|\int_{0}^{t}(\tau+1)^{-1}|\varphi(\tau)|^{2 / n} d \tau-|\varphi(t)|^{2 / n} \log (t+1)-\Psi_{+}\right\|_{\mathbf{L}^{\infty}} \\
\leq C \rho^{(2 / n+2)(2 / n)}(t+1)^{(-2 / n)(\delta-\gamma)} \log (t+1)
\end{gathered}
$$

and

$$
\begin{gathered}
\left\|\int_{0}^{t}(\tau+1)^{-1}|\varphi(\tau)|^{2 / n} d \tau-|\varphi(t)|^{2 / n} \log (t+1)-\Psi_{+}\right\|_{\dot{\mathbf{H}}^{\beta}} \\
\leq C \rho^{4 / n+1}(t+1)^{(-1 / 2)(\sigma-\beta)+\gamma} \log (t+1) .
\end{gathered}
$$

Hence

$$
\begin{gathered}
\left\|\int_{0}^{t}(\tau+1)^{-1}|\varphi(\tau)|^{2 / n} d \tau-\left|y_{+}\right|^{2 / n} \log (t+1)-\Psi_{+}\right\|_{\mathbf{L}^{\infty}} \\
\leq C \rho^{(2 / n+2)(2 / n)}(t+1)^{(-2 / n)(\delta-\gamma)} \log (t+1)
\end{gathered}
$$

and

$$
\begin{gathered}
\left\|\int_{0}^{t}(\tau+1)^{-1}|\varphi(\tau)|^{2 / n} d \tau-\left|y_{+}\right|^{2 / n} \log (t+1)-\Psi_{+}\right\|_{\dot{\mathbf{H}}^{\beta}} \\
\leq C \rho^{4 / n+1}(t+1)^{(-1 / 2)(\sigma-\beta)+\gamma} \log (t+1) .
\end{gathered}
$$

We have

$$
\begin{aligned}
\| \varphi(t)- & e^{-i \lambda\left|y_{+}\right|^{2 / n} \log (t+1)-i \lambda \Psi_{+}} y_{+} \|_{\mathbf{L}^{\infty}} \\
= & \left\|e^{-i \lambda \int_{0}^{t}(\tau+1)^{-1}|\varphi(\tau)|^{2 / n} d \tau} y(t)-e^{-i \lambda\left|y_{+}\right|^{2 / n} \log (t+1)-i \lambda \Psi_{+}} y_{+}\right\|_{\mathbf{L}^{\infty}} \\
= & \| e^{-i \lambda \int_{0}^{t}(\tau+1)^{-1}|\varphi(\tau)|^{2 / n} d \tau}\left(y(t)-y_{+}\right) \\
& +\left(e^{-i \lambda \int_{0}^{t}(\tau+1)^{-1}|\varphi(\tau)|^{2 / n} d \tau}-e^{-i \lambda\left|y_{+}\right|^{2 / n} \log (t+1)-i \lambda \Psi_{+}}\right) y_{+} \|_{\mathbf{L}^{\infty}} \\
\leq & \left\|y(t)-y_{+}\right\|_{\mathbf{L}^{\infty}} \\
& +C\left\|\int_{0}^{t}(\tau+1)^{-1}|\varphi(\tau)|^{2 / n} d \tau-\left|y_{+}\right|^{2 / n} \log (t+1)-\Psi_{+}\right\|_{\mathbf{L}^{\infty}}\left\|y_{+}\right\|_{\mathbf{L}^{\infty}} .
\end{aligned}
$$

We apply Lemma 4.1 and (4.4) to the right hand side of the above to find

$$
\begin{aligned}
\| \varphi(t) & -e^{-i \lambda\left|y_{+}\right|^{2 / n} \log (t+1)-i \lambda \Psi_{+}} y_{+} \|_{\mathbf{L}^{\infty}} \\
& \leq C \rho^{2 / n+2}(t+1)^{-(\delta-\gamma)}+C \rho^{(2 / n+2)(2 / n)+1}(t+1)^{(-2 / n)(\delta-\gamma)} \log (t+1) .
\end{aligned}
$$

We let $\widehat{u_{+}}=e^{-i \lambda \Psi_{+}} y_{+} \in \mathbf{L}^{\infty} \cap \dot{\mathbf{H}}^{\beta}$. Then we get

$$
\left\|\varphi(t)-\widehat{u_{+}} e^{-i \lambda\left|\widehat{u_{+}}\right|^{2 / n} \log (t+1)}\right\|_{\mathbf{L}^{\infty}} \leq C \rho^{(2 / n+2)(2 / n)+1}(t+1)^{(-2 / n)(\delta-\gamma)} \log (t+1) .
$$

Similarly, we have

$$
\left\|\varphi(t)-\widehat{u_{+}} e^{-i \lambda\left|\widehat{u_{+}}\right|^{2 / n} \log (t+1)}\right\|_{\dot{\mathbf{H}}^{\beta}} \leq C \rho^{4 / n+2}(t+1)^{(-1 / 2)(\sigma-\beta)+\gamma} \log (t+1) .
$$


This is the second estimates of the lemma. By the factorization formula $u(t)=M(t+$ 1) $D_{t+1} \mathcal{V}(t+1) \varphi$ we obtain

$$
\begin{aligned}
u(t) & -M(t+1) D_{t+1}\left(\widehat{u_{+}} e^{-i \lambda\left|\widehat{u_{+}}\right|^{2 / n} \log (t+1)}\right) \\
& =M(t+1) D_{t+1}(\mathcal{V}(t+1)-1) \varphi+M(t+1) D_{t+1}\left(\varphi(t)-\widehat{u_{+}} e^{-i \lambda\left|\widehat{u_{+}}\right|^{2 / n} \log (t+1)}\right) .
\end{aligned}
$$

By (2.1) and (4.6) we find

$$
\begin{aligned}
\| u(t) & -M(t+1) D_{t+1} \widehat{u_{+}} e^{-i \lambda\left|\widehat{u_{+}}\right|^{2 / n} \log (t+1)} \|_{\mathbf{L}^{\infty}} \\
& \leq C \rho^{2}(t+1)^{-n / 2-(\delta-\gamma)}+C \rho^{(2 / n+2)(2 / n)+1}(t+1)^{-n / 2-(2 / n)(\delta-\gamma)} \log (t+1)
\end{aligned}
$$

which implies the first estimates of the lemma.

Acknowledgments. We would like to thank unknown referee for useful comments.

\section{References}

[1] H. Bahouri, J.-Y. Chemin and R. Danchin, Fourier analysis and nonlinear partial differential equations, Springer, Berlin, 2011.

[2] R. Carles, Geometric optics and long range scattering for one dimensional nonlinear Schrödinger equations, Commun. Math. Phys., 220 (2001), 41-67.

[3] Y. Cho and T. Ozawa, On the semirelativistic Hartree-type equation, SIAM J. Math. Anal., 38 (2006), 1060-1074.

[4] J.-M. Delort, Existence globale et comportement asymptotique pour l'équation de Klein-Gordon quasi-linéaire à données petites en dimension 1, Ann. Sci. École Norm. Sup. (4), 34 (2001), 1-61.

[5] J. Ginibre and T. Ozawa, Long range scattering for nonlinear Schrödinger and Hartree equations in space dimension $n \geq 2$, Commun. Math. Phys., 151 (1993), 619-645.

[6] N. Hayashi, C. Li and P. I. Naumkin, Nonlinear Schrödinger systems in 2d with nondecaying final data, J. Differential Equations, 260 (2016), 1472-1495.

[ 7 ] N. Hayashi and P. I. Naumkin, Asymptotics in large time of solutions to nonlinear Schrödinger and Hartree equations, Amer. J. Math., 120 (1998), 369-389.

[8] N. Hayashi and P. I. Naumkin, Domain and range of the modified wave operator for Schrödinger equations with a critical nonlinearity, Commun. Math. Phys., 267 (2006), 477-492.

[ 9 ] N. Hayashi and P. I. Naumkin, The initial value problem for the cubic nonlinear Klein-Gordon equation, Zeitschrift fur Angewandte Mathematik und Physik, 59 (2008), 1002-1028.

[10] N. Hayashi and P. I. Naumkin, Final state problem for the cubic nonlinear Klein-Gordon equation, J. Math. Phys., 50 (2009), 103511, 14pp.

[11] T. Kato, On nonlinear Schrödinger equations II, $H^{s}$-solutions and unconditional wellposedness, J. Anal. Math., 67 (1995), 281-306.

[12] C. Li and N. Hayashi, Critical nonlinear Schrödinger equations with data in homogeneous weighted $\mathbf{L}^{2}$ spaces, J. Math. Anal. Appl., 419 (2014), 1214-1234.

[13] S. Masaki and J. Segata, Modified scattering for the quadratic nonlinear Klein-Gordon equation in two space dimensions, preprint, 2016.

[14] T. Ozawa, Long range scattering for nonlinear Schrödinger equations in one space dimension, Commun. Math. Phys., 139 (1991), 479-493. 
Nakao HAYASHI

Department of Mathematics

Graduate School of Science

Osaka University

Toyonaka

Osaka 560-0043, Japan

E-mail: nhayashi@math.sci.osaka-u.ac.jp
Chunhua LI

Department of Mathematics

College of Science

Yanbian University

No.977 Gongyuan Road, Yanji City Jilin Province 133002, China

E-mail: sxlch@ybu.edu.cn

Pavel I. NAumkin

Centro de Ciencias Matemáticas

UNAM Campus Morelia

AP 61-3 (Xangari)

Morelia CP 58089

Michoacán, Mexico

E-mail: pavelni@matmor.unam.mx 